\title{
Edge-partitioning a graph into paths: beyond the Barát-Thomassen conjecture*
}

\author{
Julien Bensmail $^{a}$, Ararat Harutyunyan ${ }^{b}$, \\ Tien-Nam Le ${ }^{c}$, and Stéphan Thomasséc \\ ${ }^{a}$ I3S and INRIA \\ Université Nice-Sophia-Antipolis \\ 06900 Sophia-Antipolis, France \\ ${ }^{b}$ LAMSADE, CNRS \\ Université Paris-Dauphine, PSL Research University \\ 75016 Paris, France \\ ${ }^{c}$ Laboratoire d'Informatique du Parallélisme \\ École Normale Supérieure de Lyon \\ 69364 Lyon Cedex 07, France
}

\begin{abstract}
In 2006, Barát and Thomassen conjectured that there is a function $f$ such that, for every fixed tree $T$ with $t$ edges, every $f(t)$-edgeconnected graph with its number of edges divisible by $t$ has a partition of its edges into copies of $T$. This conjecture was recently verified by the current authors and Merker [1].

We here further focus on the path case of the Barát-Thomassen conjecture. Before the aforementioned general proof was announced, several successive steps towards the path case of the conjecture were made, notably by Thomassen [11, 12, 13], until this particular case was totally solved by Botler, Mota, Oshiro and Wakabayashi [2]. Our goal in this paper is to propose an alternative proof of the path case with a weaker hypothesis: Namely, we prove that there is a function $f$ such that every 24-edge-connected graph with minimum degree $f(t)$ has an edge-partition into paths of length $t$ whenever $t$ divides the number of edges. We also show that 24 can be dropped to 4 when the graph is eulerian.
\end{abstract}

${ }^{*}$ The first author was supported by ERC Advanced Grant GRACOL, project no. 320812. The second author was supported by an FQRNT postdoctoral research grant and CIMI research fellowship. The fourth author was partially supported by the ANR Project STINT under Contract ANR-13-BS02-0007. 


\section{Introduction}

Unless stated otherwise, graphs considered here are generally simple, loopless and undirected. Given a graph $G$, we denote by $V(G)$ and $E(G)$ its vertex and edge sets, respectively. Given a vertex $v$ of $G$, we denote by $d_{G}(v)$ (or simply $d(v)$ in case no ambiguity is possible) the degree of $v$ in $G$, i.e., the number of edges incident to $v$ in $G$. We denote by $\delta(G)$ and $\Delta(G)$ the minimum and maximum, respectively, degree of a vertex in $G$. When $X$ is a subset of vertices of $G$, we denote by $d_{X}(v)$ the degree of $v$ in the subgraph of $G$ induced by $X \cup\{v\}$. Given two graphs $G=(V, E)$ and $H=(V, F)$ with $F \subseteq E$, we denote by $G \backslash H$ the graph $(V, E \backslash F)$.

Let $G$ and $H$ be two graphs such that $|E(H)|$ divides $|E(G)|$. We say that $G$ is $H$-decomposable if there exists a partition $E_{1} \cup E_{2} \cup \ldots \cup E_{k}$ of $E(G)$ such that every $E_{i}$ induces an isomorphic copy of $H$. We then call $E_{1} \cup E_{2} \cup \ldots \cup E_{k}$ an $H$-decomposition of $G$.

This paper is devoted to the following conjecture raised by Barát and Thomassen in [4], stating that highly edge-connected graphs can be decomposed into copies of any tree.

Conjecture 1.1. For any fixed tree $T$, there is an integer $c_{T}$ such that every $c_{T}$-edge-connected graph with its number of edges divisible by $|E(T)|$ can be T-decomposed.

Conjecture 1.1 was recently solved by the current authors and Merker in [1]. For a summary of the progress towards the conjecture, we hence refer the interested reader to that paper. Before this proof was announced, the path case of the conjecture had been tackled through successive steps. First, the conjecture was verified for paths of small length, namely for $T$ being $P_{3}$ and $P_{4}$ by Thomassen $[11,12]$, where $P_{\ell}$ here and further denotes the path on $\ell$ edges. Thomassen then proved, in [13], the conjecture for arbitrarily long paths of the form $P_{2^{k}}$. Later on, Botler, Mota, Oshiro and Wakabayashi proved the conjecture for $P_{5}$ [3] before generalizing their arguments and settling the conjecture for all paths [2].

Conjecture 1.1 being now solved, many related lines of research sound quite appealing. One could for example wonder, for any fixed tree $T$, about the least edge-connectivity guaranteeing the existence of $T$-decompositions. We note that the proof of Conjecture 1.1 from [1], because essentially probabilistic, provides a huge bound on the required edge-connectivity, which is clearly far from optimal. Another interesting line of research, is about the true importance of large edge-connectivity over large minimum degree in the statement of Conjecture 1.1. Of course, one can notice that, to necessarily admit $T$-decompositions, graphs among some family must meet a least edge-connectivity condition. We however believe that this condition can be lowered a lot, provided this is offset by a large minimum degree condition. 
More precisely, we believe the following refinement of Conjecture 1.1 makes sense.

Conjecture 1.2. There is a function $f$ such that, for any fixed tree $T$ with maximum degree $\Delta_{T}$, every $f\left(\Delta_{T}\right)$-edge-connected graph with its number of edges divisible by $|E(T)|$ and minimum degree at least $f(|E(T)|)$ can be T-decomposed.

In this paper, we make a first step towards Conjecture 1.2 by showing it to hold when $\Delta_{T} \leq 2$, that is for the cases where $T$ is a path.

Theorem 1.3. For every integer $\ell \geq 2$, there exists $d_{\ell}$ such that every 24edge-connected graph $G$ with minimum degree at least $d_{\ell}$ has a decomposition into paths of length $\ell$ and a path of length at most $\ell$.

In particular, our proof of Theorem 1.3 yields a third proof of the path case of Conjecture 1.1. It is also important mentioning that this proof is, in terms of approach, quite different from the one from [2].

Let us, as well, again emphasize that the main point in the statement of Theorem 1.3 is that the required edge-connectivity, namely 24, is constant and not dependent on the path length $\ell$. Concerning the optimal value as $f(2)$ mentioned in Conjecture 1.2 (which is bounded above by 24, following Theorem 1.3), a lower bound on it is 3 as there exist 2-edge-connected graphs with arbitrarily large minimum degree admitting no $P_{\ell^{-}}$decomposition for some $\ell$. To be convinced of this statement, just consider the following construction. Start from the 2-edge-connected graph $G$ depicted in Figure 1, which admits no $P_{9}$-decomposition. To now obtain a 2 -edge-connected graph with arbitrarily large minimum degree $d$ from it, just consider any 2-edgeconnected graph $H$ with sufficiently large minimum degree (i.e., at least $d$ ) and verifying $|E(H)| \equiv 7(\bmod 9)$. Then consider any vertex $v$ of $G$ with small degree, and add two edges from $v$ to a new copy of $H$. Repeating this transformation as long as necessary, we get a new graph which is still 2-edge-connected, with minimum degree at least $d$ and whose size is a multiple of 9 (due to the size of $G$ and $H$ ), but with no $P_{9}$-decomposition otherwise, it can be easily checked that $G$ would admit a $P_{9}$-decomposition, a contradiction.

Very roughly, the proof of Theorem 1.3 goes as follows. When the graph $G$ has an eulerian tour $\mathcal{E}$, a natural strategy to obtain a $P_{\ell \text {-decomposition of }}$ $G$ is to cut $\mathcal{E}$ into consecutive $\ell$-paths. Of course we may be unsuccessful in doing so since several consecutive edges of $\mathcal{E}$ may be conflicting, that is have common vertices, hence inducing a cycle. Note however that if every edge of $\mathcal{E}$ (and hence of $G$ ) is already a path of length at least $\ell$, then, cutting pieces along $\mathcal{E}$, only its consecutive paths can be conflicting - hence bringing the notion of conflict to a very local setting. Following this easy idea, the proof consists in expressing $G$ as a $(\geq \ell)$-path-graph (i.e., a system of edge-disjoint 


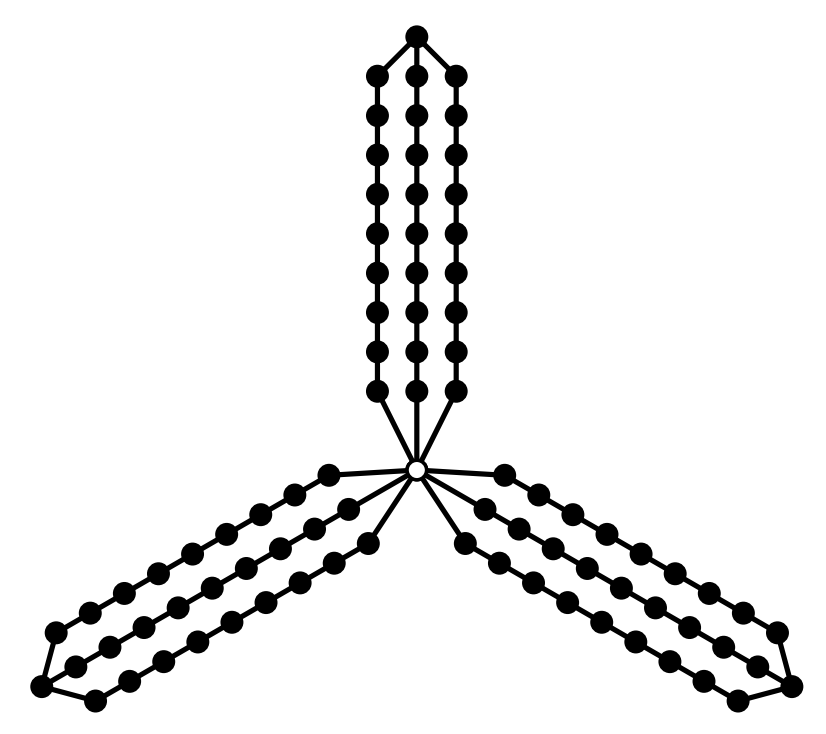

Figure 1: Part of the construction for obtaining 2-edge-connected graphs with arbitrarily large minimum degree but no $P_{\ell^{-}}$-decomposition for some $\ell$.

paths of length at least $\ell$ covering all edges) $H$ with low conflicts between its paths, then making $H$ eulerian somehow while keeping low conflicts, and eventually deducing a conflictless eulerian tour that can eventually be safely cut into $\ell$-paths.

One side fact resulting from our proof scheme is that when $G$ is eulerian, making $H$ eulerian requires less edge-connectivity. This remark, and additional arguments, allow us to also prove the following result.

Theorem 1.4. For every integer $\ell \geq 2$, there exists $d_{\ell}$ such that every 4-edge-connected eulerian graph with minimum degree at least $d_{\ell}$ has a decomposition into paths of length $\ell$ and a path of length at most $\ell$.

This paper is organized as follows. We start by introducing and recalling preliminary tools and results in Section 2. The notion of path-graphs and some properties of these objects are then introduced in Section 3. Particular path-graphs, which we call path-trees, needed to repair eulerianity of pathgraphs are then introduced and studied in Section 4. With all notions and results in hands, we then prove Theorems 1.3 and 1.4 in Section 5.

\section{Tools and preliminary results}

Let $H=(V, F)$ be a spanning subgraph of a graph $G=(V, E)$. Let $\alpha$ be some real number in $[0,1]$. We say that $H$ is $\alpha$-sparse in $G$ if $d_{H}(v) \leq$ $\alpha d_{G}(v)$ for all vertices $v$ of $G$. Conversely, we say that $H$ is $\alpha$-dense in $G$ if $d_{H}(v) \geq \alpha d_{G}(v)$ for all vertices $v$ of $G$. We will also heavily depend 
on subgraphs of $G$ which are both (roughly) $\alpha$-sparse and $\alpha$-dense. This definition depends on the length of the path $\ell$. We say that $H$ is an $\alpha$ fraction of $G$ if $\alpha d_{G}(v)-10 \ell^{\ell} \leq d_{H}(v) \leq \alpha d_{G}(v)+10 \ell^{\ell}$.

Given an (improper) edge-coloring $\phi$ of some graph $G$ and a color $i$, for every vertex $v$ of $G$ we denote by $d_{i}(v)$ the number of $i$-colored edges incident to $v$. We call $\phi$ nearly equitable if, for every vertex $v$ and every pair of colors $i \neq j$, we have $\left|d_{i}(v)-d_{j}(v)\right| \leq 2$. We can now recall a result of de Werra (cf. [10], Theorem 8.7), and its corollary concerning $1 / k$-fractions.

Proposition 2.1. Let $k \geq 1$. Every graph has a nearly equitable improper $k$-edge-coloring.

Proposition 2.2. Let $k \geq 1$. Every graph $G=(V, E)$ has a subgraph $H=(V, F)$ such that $\left|d_{H}(v)-d_{G}(v) / k\right| \leq 2$ for every vertex $v$.

We now recall three results on oriented graphs. The first of these is a folklore result on balanced orientations of graphs.

Proposition 2.3. Every multigraph $G$ has an orientation $D$ such that $\left|d_{D}^{-}(v)-d_{D}^{+}(v)\right| \leq 1$ for every vertex $v$.

The proof is straightforward. We first arbitrarily pair vertices of odd degree of $G$, then add a dummy edge between every pair to obtain a multigraph $G^{\prime}$ in which every vertex has even degree. Orienting the edges of $G^{\prime}$ as they are encountered when going along an eulerian tour, we then deduce an orientation $D^{\prime}$ of $G^{\prime}$ such that $d_{D^{\prime}}^{-}(v)=d_{D^{\prime}}^{+}(v)$ for every vertex $v$. Removing the dummy edges results in a desired orientation $D$ of $G$.

The second result is a result of Nash-Williams (see [9]) implying that any graph with large edge-connectivity admits a balanced orientation with large arc-connectivity. In the following, a digraph $D$ is $k$-arc-strong if the removal of any set of at most $k-1$ arcs leaves $D$ strongly-connected.

Proposition 2.4. Every $2 k$-edge-connected multigraph has an orientation $D$ such that $D$ is $k$-arc-strong and such that $\left|d^{-}(v)-d^{+}(v)\right| \leq 1$ for every vertex $v$.

The third result we recall is due to Edmonds (see [5]) and expresses a condition for a digraph to admit many arc-disjoint rooted arborescences. In the statement, an out-arborescence of a digraph $D$ refers to a rooted spanning tree $T$ of $D$ whose arcs are oriented in such a way that the root has in-degree 0 , and every other vertex has in-degree 1.

Proposition 2.5. A directed multigraph with a special vertex $z$ has $k$ arcdisjoint out-arborescences rooted at $z$ if and only if the number of arc-disjoint paths between $z$ and any vertex is at least $k$.

We end this section recalling probabilistic tools we will need in the next sections (refer e.g., to [8] for more details). The first of these is the wellknown Local Lemma. 
Proposition 2.6 (Lovász Local Lemma). Let $A_{1}, \ldots, A_{n}$ be a finite set of events in some probability space $\Omega$, with $\mathbb{P}\left[A_{i}\right] \leq p$ for all $i$. Suppose that each $A_{i}$ is mutually independent of all but at most $d$ other events $A_{j}$. If $4 p d<1$, then $\operatorname{Pr}\left[\cap_{i=1}^{n} \overline{A_{i}}\right]>0$.

We will also require the use of the following concentration inequality due to McDiarmid [7] (see also [8]) concerning random permutations. We think of a permutation as a bijective function. In what follows, a choice is defined to be the position that a particular element gets mapped to in a permutation.

Proposition 2.7 (McDiarmid's Inequality (simplified version)). Let $X$ be a non-negative random variable, not identically 0 , which is determined by $m$ independent permutations $\Pi_{1}, \ldots, \Pi_{m}$. If there exist $d, r>0$ such that

- interchanging two elements in any one permutation can affect $X$ by at most d, and

- for any $s>0$, if $X \geq s$ then there is a set of at most rs choices whose outcomes certify that $X \geq s$,

then for any $0 \leq \lambda \leq \mathbb{E}[X]$,

$$
\mathbb{P}[|X-\mathbb{E}[X]|>\lambda+60 d \sqrt{r \mathbb{E}[X]}] \leq 4 e^{-\frac{\lambda^{2}}{8 d^{2} r \mathbb{E}[X]}}
$$

\section{Path-graphs}

Let $G=(V, E)$ be a graph. A path-graph $H$ on $G$ is a couple $(V, \mathcal{P})$ where $\mathcal{P}$ is a set of edge-disjoint paths of $G$. The graph $\underline{H}=(V, F)$, where $F$ contains the edges of paths in $\mathcal{P}$, is called the underlying graph of $H$. If $F=E$, then $H$ is called a path-decomposition of $G$. Two edge-disjoint paths of $G$ sharing an end $v$ are said conflicting if they also intersect in another vertex different from $v$. Equivalently, we say that two paths of $H$ issued from a same vertex are conflicting if the corresponding paths in $\underline{H}$ are conflicting.

We denote by $\tilde{H}$ the multigraph on vertex set $V$ and edge set the multiset containing a pair $u v$ for each path from $u$ to $v$ in $\mathcal{P}$ (if $\mathcal{P}$ contains several paths from $u$ to $v$, we add as many edges $u v$ ). We now transfer the usual definitions of graphs to path-graphs. The degree of a vertex $v$ in $H$, denoted $d_{H}(v)$, is the degree (with multiplicity) of $v$ in $\tilde{H}$. We say that $H$ is connected if $\tilde{H}$ is connected, that $H$ is eulerian if $\tilde{H}$ is eulerian, and that $H$ is a pathtree if $\tilde{H}$ is a tree (even if the paths of $\mathcal{P}$ pairwise intersect). From a tour in $\tilde{H}$, we naturally get a corresponding tour in $H$. Such a tour is said non-conflicting if every two of its consecutive paths are non-conflicting.

We need also to speak of the lengths of the paths in $\mathcal{P}$. Let us say that $H$ is an $\ell$-path-graph if all paths in $\mathcal{P}$ have length $\ell$, a $(\geq \ell)$-path-graph if all 
paths in $\mathcal{P}$ have length at least $\ell$, an $\left(\ell_{1}, \ell_{2}, \ldots\right)$-path-graph if all paths in $\mathcal{P}$ have length among $\left\{\ell_{1}, \ell_{2}, \ldots\right\}$, and an $[\ell, \ell+i]$-path-graph if all paths in $\mathcal{P}$ have length in the interval $[\ell, \ell+i]$.

In general, the paths of a path-graph $H=(V, \mathcal{P})$ can pairwise intersect, and we would hence like to measure how much. For every vertex $v$, let $\mathcal{P}_{H}(v)$ be the set of paths in $H$ incident with $v$, i.e., starting or ending at $v$. The conflict ratio of $v$ is

$$
\operatorname{conf}(v):=\frac{\max _{w \neq v}\left|\left\{P \in \mathcal{P}_{H}(v): w \in P\right\}\right|}{d_{H}(v)} .
$$

Now, regarding $H$, we set $\operatorname{conf}_{G}(H):=\max _{v} \operatorname{conf}(v)$. When the graph $G$ is clear from the context, we will often omit the subscript in the notation. Clearly we always have $\operatorname{conf}(H) \leq 1$.

With all the terminology above in hand, we can now prove (or recall) properties of path-graphs. We start by recalling that, as desired, eulerian path-graphs with somewhat low conflicts have non-conflicting eulerian tours. This matter was actually already considered by Jackson (cf. [6], Theorem 6.3) under the following different terminology.

For a vertex $v$, let $E_{v}$ be the set of edges incident to $v$. A generalised transition system $S$ for a graph $G$ is a set of functions $\left\{S_{v}\right\}_{v \in V(G)}$ such that $S_{v}: E_{v} \rightarrow 2^{E_{v}}$ and whenever $e_{1} \in S_{v}\left(e_{2}\right)$, we have that $e_{2} \in S_{v}\left(e_{1}\right)$. We say that an eulerian tour $\mathcal{E}$ is compatible with $S$ if for all $v \in V(G)$, whenever $e_{1} \in S_{v}\left(e_{2}\right)$ it follows that $e_{1}$ and $e_{2}$ are not consecutive edges in $\mathcal{E}$.

Theorem 3.1 (Jackson [6]). Let $S$ be a generalised transition system for an eulerian graph $G$. Suppose that for each vertex $v \in V(G)$ and $e \in E_{v}$, we have

(i) $\left|S_{v}(e)\right| \leq \frac{1}{2} d(v)-1$ when $d(v) \equiv 0(\bmod 4)$ or $d(v)=2$, and

(ii) $\left|S_{v}(e)\right| \leq \frac{1}{2} d(v)-2$, otherwise.

Then $G$ has an eulerian tour compatible with $S$.

From Theorem 3.1, the following result is immediate.

Theorem 3.2. Every eulerian $[\ell, \ell+3]$-path-graph $H$ with $\operatorname{conf}(H) \leq 1 / 2(\ell+$ 10) and $d_{H}(v) \geq \ell+10$ has a non-conflicting eulerian tour.

Proof. Let $P \in \mathcal{P}_{H}(v)$. The number of paths of $\mathcal{P}_{H}(v)$ conflicting with $P$ is at most $\frac{1}{2(\ell+10)}(\ell+3) d_{H}(v)$, and so at most $\frac{1}{2} d_{H}(v)-2$ since $d_{H}(v) \geq \ell+10$. The result now follows from Theorem 3.1.

We now prove that every graph with large enough minimum degree can be expressed as a $(\geq \ell)$-path-graph meeting particular properties. 
Theorem 3.3. Let $\ell$ be a positive integer, and $\varepsilon$ be an arbitrarily small positive real number. There exists $L$ such that if $G=(V, E)$ is a graph with minimum degree at least $L$, then there is an $\ell$-path-graph $H$ on $G$ with

- $\operatorname{conf}(H) \leq \varepsilon$,

- $d_{H}(v) / d_{G}(v) \in\left[\frac{1-\varepsilon}{\ell}, \frac{1+\varepsilon}{\ell}\right]$ for every vertex $v$, and

- $d_{G \backslash \underline{H}}(v) \leq \varepsilon d_{H}(v)$ for every vertex $v$.

Proof. Let $c:=[\sqrt{L}]$ and $b:=\left[c^{2 / 3}\right]$, and pick $L$ so that $b \gg \ell$. According to Proposition 2.1, we can nearly equitably color the edges of $G$ with $\ell$ colors. For every color $i$, applying Proposition 2.3 we can orient the $i$-colored edges so that the numbers of in-edges and out-edges of color $i$ incident to every vertex $v$ differ by at most 1 . Let $E_{i}^{-}(v)$ and $E_{i}^{+}(v)$ be the sets of $i$-colored in-edges and out-edges, respectively, incident to $v$. Then, for every color $i \in\{1, \ldots, \ell-1\}$, we have

$$
|| E_{i}^{-}(v)|-| E_{i+1}^{+}(v)|| \leq 2 .
$$

For the sake of convenience, we would like to have that $\left|E_{i}^{-}(v)\right|=\left|E_{i+1}^{+}(v)\right|$ for all $i$ and $v$. To this end, we add a dummy vertex $v_{0}$ to $G$. Now, if $\left|E_{i}^{-}(v)\right|-\left|E_{i+1}^{+}(v)\right|=k>0$, then we add $k$ dummy edges of color $i+1$ from $v$ to $v_{0}$ to equalize $\left|E_{i}^{-}(v)\right|$ and $\left|E_{i+1}^{+}(v)\right|$. Similarly, if $\left|E_{i+1}^{+}(v)\right|-\left|E_{i}^{-}(v)\right|=$ $k>0$, then we add $k$ dummy edges of color $i$ from $v_{0}$ to $v$.

Now, for every $v \in V(G)$ and color $i \in\{1, \ldots, \ell\}$, we choose $r_{v, i} \in$ $\{0, \ldots, c-2\}$ such that $E_{i}^{-}(v) \equiv r_{v, i}(\bmod c-1)$. Since the minimum degree in each color in $G$ is greater than $c(c-2)$, we can partition every set $E_{i}^{-}(v)$ into subsets of size $c$ and $c-1$ so that precisely $r_{v, i}$ of them have size $c$. As $E_{i+1}^{+}(v)=E_{i}^{-}(v)$, we can similarly partition every set $E_{i+1}^{+}(v)$ into subsets of size $c$ and $c-1$ so that precisely $r_{v, i}$ of them have size $c$.

We call these subsets of edges $i$-half cones and $(i+1)$-half cones, respectively. Now, for each vertex $v$ and color $i, 1 \leq i \leq \ell-1$, we arbitrarily pair $i$-half cones of $E_{i}^{-}(v)$ with $(i+1)$-half cones $E_{i+1}^{+}(v)$ in a way such that in each pair the size of the two half cones are equal. We call such a pair an $i$-cone at vertex $v$. Thus, an $i$-cone $\varphi$ at some vertex $v$ consists of an $i$-half cone $\varphi^{-}$and an $(i+1)$-half cone $\varphi^{+}$with $\left|\varphi^{-}\right|=\left|\varphi^{+}\right|$. Note that an edge $e$ of color $i$ directed from a vertex $u$ to a vertex $v$ in $G$ appears both in an $i$-half cone of $E_{i}^{+}(u)$ as well as in an $i$-half cone of $E_{i}^{-}(v)$, but we do not require these two $i$-half cones to have the same size. By convention, we do not create a cone at the dummy vertex $v_{0}$. However, each edge $u v_{0}$ will still be inside a cone at vertex $u$. We also remark that the 1-half cones of $E_{1}^{+}(v)$ and the $\ell$-half cones of $E_{\ell}^{-}(v)$ do not get paired with other half cones. Nevertheless, we will adopt the convention that whenever we talk of a general cone $\varphi$, we will assume that $\varphi$ might also consist of a single 1-half cone or $\ell$-half cone of the aforementioned type. 
We now have a fixed set of cones on $G$. To obtain our desired pathgraph, we will use the cone structure to construct rainbow paths of length $\ell$, i.e., paths where for all $i$ the $i^{t h}$ edge of every path is of color $i$. One way to obtain this is to randomly match edges of the two half cones of every cone. Indeed, this is what we do. For each cone $\varphi$ we carry out random permutations $\pi_{\varphi}^{-}$of the edges of $\varphi^{-}$and $\pi_{\varphi}^{+}$of the edges of $\varphi^{+}$. We then pair the edges $\pi_{\varphi}^{-}(k)$ and $\pi_{\varphi}^{+}(k)$ for each $1 \leq k \leq c$. If $\varphi$ is actually a special 1-half cone or $\ell$-half cone, then there is only one random permutation performed at $\varphi$, which will have no effect on the decomposition as will be apparent shortly. Note that each edge $e=u v$ of $G$, with the exception of some edges of 1-cones, some edges of $\ell$-cones and the dummy edges, is in exactly two cones - one centered at $u$ and the second centered at $v$. Thus, $e$ is involved in two random permutations corresponding to the two permutations of the two half cones containing it. Therefore, given the random matchings, each non-dummy edge $e=u v$ of color $i, 1<i<\ell$, is paired exactly with one edge of color $i-1$ (which enters $u$ ) and one edge of color $i+1$ (which exits $v$ ). From an arbitrary edge, we can thus go forward and backward by edges paired with it until we reach edges of color $\ell$ or 1 (unless we reach dummy edges). Thus, the random matchings yield a natural decomposition of all edges of $G$ into edge-disjoint walks. Unfortunately, some of the walks will not be paths. We will divide the walks into three types. Of the first type are those walks which are paths, and thus by construction they are necessarily isomorphic to $P_{\ell}$. A walk that is not a path and which does not use the dummy vertex $v_{0}$ is called a bad walk; note that every bad walk is of length $\ell$. A walk that uses the dummy vertex $v_{0}$ is called a short walk. Note that a short walk is no longer extended from $v_{0}$ as there is no cone centered at $v_{0}$.

For each cone $\varphi$, there are $c-1$ or $c$ walks via $\varphi$, depending on $|\varphi|$. We will show that, with high probability, the number of bad or short walks via $\varphi$ is negligible compared to $c$. We then will argue that proving this statement for all the cones is sufficient for us to extract a dense path-graph from $G$.

Denote $P_{\ell}:=x_{0} x_{1} \ldots x_{\ell}$. We first focus on bad walks. Suppose that $\varphi$ is a $k$-cone at some vertex $v$, and $i, j$ are two colors. We say that a bad walk $P=u_{0} u_{1} \ldots u_{\ell}$ going through $\varphi$ is $(i, j)$-bad if its $i^{\text {th }}$ vertex and $j^{\text {th }}$ vertex are the same, that is, $u_{j}=u_{i}$. Let $A_{\varphi}(i, j)$ be the event that the number of $(i, j)$-bad walks going through the cone $\varphi$ is greater than $b$. We will show that $\mathbb{P}\left[A_{\varphi}(i, j)\right]<4 e^{-c^{2 / 3} / 64}$.

Denote by $P_{i, k}$ and $P_{j, k}$ the subpaths from $x_{i}$ to $x_{k}$, and $x_{j}$ to $x_{k}$ in $P_{\ell}$, respectively. In case one of these paths is contained in another, we may assume that $P_{i, k}$ is contained in $P_{j, k}$. Let $x_{j^{\prime}}$ be the neighbor of $x_{j}$ in $P_{j, k}$. Note that $j^{\prime} \in\{j-1, j+1\}$. Let $\mathcal{P}_{\varphi}$ be the set of walks that go through $\varphi$ which are not short. Clearly, $\left|\mathcal{P}_{\varphi}\right| \leq c$.

We define $\Omega_{j^{\prime}}$ to be the set of all $j^{\prime}$-cones in $G$ if $j^{\prime}=j-1$, and the set of all $j$-cones if $j^{\prime}=j+1$. Let $\Pi$ be an arbitrary but fixed outcome 
of all permutations at all cones except the set of permutations on $\Omega_{j^{\prime}}$. In other words, given $\Pi$, we only need to know the outcomes of the set of permutations $\left\{\pi_{\varphi}^{+}, \pi_{\varphi}^{-} \mid \varphi \in \Omega_{j^{\prime}}\right\}$ to know the decomposition of the walks in $G$. We will condition on $\Pi$; that is, we will show that $\left.\mathbb{P}\left[A_{\varphi}(i, j)\right] \mid \Pi\right]<$ $4 e^{-c^{2 / 3} / 64}$ for any $\Pi$. Clearly, since $\Pi$ is arbitrary, this is sufficient to give us the uniform bound $\mathbb{P}\left[A_{\varphi}(i, j)\right]<4 e^{-c^{2 / 3} / 64}$.

Let $\mathcal{P}_{\varphi}^{\prime}$ denote the set of walks $\mathcal{P}_{\varphi}$ conditional on $\Pi$. Let $X_{\varphi}$ be the number of $(i, j)$-bad walks going through the cone $\varphi$ conditional on $\Pi$. Вy fixing $\Pi$, the set $\mathcal{P}_{\varphi}^{\prime}$ is also fixed. Indeed, each $P^{\prime} \in \mathcal{P}_{\varphi}^{\prime}$ is a partial subwalk, where we know the vertex of $P^{\prime}$ that lies in some half-cone of a cone $\psi \in$ $\Omega_{j^{\prime}}$. Note that the vertex $u_{i}$ of $P^{\prime}$ corresponding to $x_{i}$ is already known. Moreover, the vertex $u_{j^{\prime}}$ corresponding to the vertex $x_{j^{\prime}}$ is known as well.

Note that whether $P^{\prime}$ is $(i, j)$-bad depends only on the permutations $\pi_{\psi}^{-}$ and $\pi_{\psi}^{+}$. Note that there are $c-1$ or $c$ different images possible to match $u_{j^{\prime}}$ when the random permutations $\pi_{\psi}^{-}$and $\pi_{\psi}^{+}$are carried out, and only one of which could possibly be $u_{i}$. Thus, the probability that $P^{\prime}$ is $(i, j)$-bad is at most $\frac{1}{c-1}$.

Now, by linearity of expectation,

$$
\mathbb{E}\left[X_{\varphi}\right] \leq\left|\mathcal{P}_{\varphi}\right| \cdot \frac{1}{c-1} \leq \frac{c}{c-1} .
$$

We will apply McDiarmid's inequality to the random variable $Y_{\varphi}$ defined by $Y_{\varphi}:=X_{\varphi}+c^{2 / 3}$. Clearly $\mathbb{E}\left[Y_{\varphi}\right]=\mathbb{E}\left[X_{\varphi}\right]+c^{2 / 3} \in\left[c^{2 / 3}, c^{2 / 3}+2\right]$. Only the permutations $\pi_{\psi}^{-}, \pi_{\psi}^{+}$with $\psi \in \Omega_{j^{\prime}}$ affect $X_{\varphi}$ and thus $Y_{\varphi}$. If two elements in one of these permutations are interchanged, then the structure of two walks in $\mathcal{P}_{\varphi}$ changes. However, clearly the number of $(i, j)$-bad walks in $\mathcal{P}_{\varphi}$ cannot change by more than 2 . Thus, we can choose $d=2$ in McDiarmid's inequality.

If $Y_{\varphi} \geq s$, then $X_{\varphi} \geq s-c^{2 / 3}$, and thus at least $s-c^{2 / 3}$ of the walks in $\mathcal{P}_{\varphi}$ are $(i, j)$-bad. Let $P^{\prime} \in \mathcal{P}_{\varphi}^{\prime}$ be a subwalk of a walk $P$ that is counted by $X_{\varphi}$. As before, let $u_{i}=u_{j}$ denote the images of $x_{i}$ and $x_{j}$ in $P$, and $\psi \in \Omega_{j^{\prime}}$ the cone through which $P^{\prime}$ passes. To verify that $P$ is $(i, j)$-bad, we only need to reveal the two elements $\pi_{\psi}^{+}(s), \pi_{\psi}^{-}(s)$, where $1 \leq s \leq c$ is the value such that the edge $u_{j^{\prime}} u_{j} \in\left\{\pi_{\psi}^{+}(s), \pi_{\psi}^{-}(s)\right\}$.

Thus, $X_{\varphi} \geq s-c^{2 / 3}$ can be certified by the outcomes of $2\left(s-c^{2 / 3}\right)<2 s$ choices and we can choose $r=2$ in McDiarmid's inequality. By applying McDiarmid's inequality to $Y_{\varphi}$ with $\lambda=\mathbb{E}\left[Y_{\varphi}\right], d=2, r=2$, we get

$$
\mathbb{P}\left[\left|Y_{\varphi}-\mathbb{E}\left[Y_{\varphi}\right]\right|>\mathbb{E}\left[Y_{\varphi}\right]+120 \sqrt{2 \mathbb{E}\left[Y_{\varphi}\right]}\right] \leq 4 e^{-\frac{\mathbb{E}\left[Y_{\varphi}\right]}{64}} \leq 4 e^{-\frac{c^{2 / 3}}{64}}
$$

and thus $\mathbb{P}\left[X_{\varphi}>2 c^{2 / 3}\right] \leq 4 e^{-c^{2 / 3} / 64}$. So we have $\mathbb{P}\left[A_{\varphi}(i, j) \mid \Pi\right]<4 e^{-c^{2 / 3} / 64}$. Since $\Pi$ is arbitrary it follows that $\mathbb{P}\left[A_{\varphi}(i, j)\right]<4 e^{-c^{2 / 3} / 64}$. Let $A_{\varphi}$ be the 
event that there are more than $\ell^{2} b$ bad walks via $\varphi$. Then

$$
\mathbb{P}\left[A_{\varphi}\right] \leq \mathbb{P}\left[\bigcup_{\forall i, j} A_{\varphi}(i, j)\right] \leq \sum_{\forall i, j} \mathbb{P}\left[A_{\varphi}(i, j)\right]<4 \ell^{2} e^{-c^{2 / 3} / 64} .
$$

We still consider the same cone $\varphi$. For an integer $j \neq k$ and vertex $u$, let $B_{\varphi}(j, u)$ be the event that the number of walks via $\varphi$, which maps $x_{j}$ to $u$, is greater than $b$, and let $B_{\varphi}(u)$ be the event that the number of walks of $\varphi$ containing $u$ is greater than $\ell b$.

We show that $\mathbb{P}\left[B_{\varphi}(j, u)\right]<4 e^{-c^{2 / 3} / 64}$. As the computation is virtually identical to the case of $\mathbb{P}\left[A_{\varphi}(i, j)\right]$, we only highlight the differences. As before, let $x_{j^{\prime}}$ be the vertex adjacent to $x_{j}$ on $P_{j, k}$, and let $\Pi$ be an arbitrary but fixed outcome of all permutations at all cones except the set of permutations on $\Omega_{j^{\prime}}$. It suffices to show that $\mathbb{P}\left[B_{\varphi}(j, u) \mid \Pi\right]<4 e^{-c^{2 / 3} / 64}$.

Let $X_{\varphi}$ denote the random variable conditional on $\Pi$ which counts the number of walks in $\mathcal{P}_{\varphi}$ where $u$ is the image of $x_{j}$. The vertex $u$ appears at most once in each cone of $\Omega_{j^{\prime}}$, so by linearity of expectation we have

$$
\mathbb{E}\left[X_{\varphi}\right] \leq\left|\mathcal{P}_{\varphi}\right| \cdot \frac{1}{c-1} \leq \frac{c}{c-1} .
$$

We again apply McDiarmid's inequality to the random variable $Y_{\varphi}$ defined by $Y_{\varphi}:=X_{\varphi}+c^{2 / 3}$. As before, $\mathbb{E}\left[Y_{\varphi}\right]=\mathbb{E}\left[X_{\varphi}\right]+c^{2 / 3}$.

Since the vertex $u$ appears at most once in each cone of $\Omega_{j^{\prime}}$, swapping two positions in any permutation of a half-cone in $\Omega_{j^{\prime}}$ can affect $X_{\varphi}$ by at most 1 . Thus, we can choose $d=1$ in McDiarmid's inequality.

If $Y_{\varphi} \geq s$, then $X_{\varphi} \geq s-c^{2 / 3}$. Let $P^{\prime}$ be a subwalk that is counted by $X_{\varphi}$. As before, we can certify that $P^{\prime}$ is counted by $X_{\varphi}$ by considering only $\psi \in \Omega_{j^{\prime}}$, the cone through which $P^{\prime}$ passes.

To certify that $P^{\prime}$ is counted by $X_{\varphi}$ we only need to reveal the two elements $\pi_{\psi}^{+}(s), \pi_{\psi}^{-}(s)$, where $s$ is the value such that one of the edges $\pi_{\psi}^{+}(s), \pi_{\psi}^{-}(s)$ contains the endpoint $u$. Thus, $X_{\varphi} \geq s-c^{2 / 3}$ can be certified by the outcomes of $2\left(s-c^{2 / 3}\right)<2 s$ choices and we can choose $r=2$ in McDiarmid's inequality. Thus, by a similar argument as above we obtain that $\mathbb{P}\left[B_{\varphi}(j, u)\right]<4 e^{-c^{2 / 3} / 64}$. Now,

$$
\mathbb{P}\left[B_{\varphi}(u)\right] \leq \mathbb{P}\left[\bigcup_{\forall i} B_{\varphi}(i, u)\right] \leq \sum_{\forall i} \mathbb{P}\left[B_{\varphi}(i, u)\right]<4 \ell e^{-c^{2 / 3} / 64}
$$

Let $B_{\varphi}$ be the event that there exists a vertex $u$ such that more than $\ell b$ walks of $\varphi$ contain $u$. The number of vertices $u$ that could possibly appear in the walks $\mathcal{P}_{\varphi}$ is at most $c+c^{2}+\ldots+c^{\ell}<c^{\ell+1}$. Hence,

$$
\mathbb{P}\left[B_{\varphi}\right]=\mathbb{P}\left[\bigcup_{\forall u} B_{\varphi}(u)\right] \leq \sum_{\forall u} \mathbb{P}\left[B_{\varphi}(u)\right]<4 c^{\ell+1} \ell e^{-c^{2 / 3} / 64} .
$$


Let $B_{\varphi}^{\prime}(j)$ be the event that the number of walks via $\varphi$ such that they enter $v_{0}$ at exactly their $j^{t h}$-vertex is greater than $b$, and let $B_{\varphi}^{\prime}$ be the event that the number of walks of $\varphi$ containing $v_{0}$ is greater than $\ell b$. We upper bound $\mathbb{P}\left[B_{\varphi}^{\prime}(j)\right]$.

The argument is virtually identical to that of the estimate above. We apply McDiarmid's inequality to the random variable $Y_{\varphi}:=X_{\varphi}+c^{2 / 3}$, where $X_{\varphi}$ is the number of walks via $\varphi$ that enter $v_{0}$ at the $j^{\text {th }}$ edge conditional on $\Pi$. As before, we obtain that $\mathbb{E}\left[X_{\varphi}\right] \leq c /(c-1), d=1, r=2$, yielding $\mathbb{P}\left[B_{\varphi}^{\prime}(j)\right] \leq 4 e^{-c^{2 / 3} / 64}$. Thus, $\mathbb{P}\left[B_{\varphi}^{\prime}\right]<4 \ell e^{-c^{2 / 3} / 64}$.

Let $J_{\varphi}=A_{\varphi} \cup B_{\varphi} \cup B_{\varphi}^{\prime}$. Then

$$
\mathbb{P}\left[J_{\varphi}\right] \leq \mathbb{P}\left[A_{\varphi}\right]+\mathbb{P}\left[B_{\varphi}\right]+\mathbb{P}\left[B_{\varphi}^{\prime}\right]<\left(\ell^{2}+c^{\ell+1} \ell+\ell\right) 4 e^{-c^{2 / 3} / 64}<e^{-b / 100} .
$$

Let $\mathcal{J}_{\varphi}$ be the set of events $J_{\psi}$ that are not mutually independent of $J_{\varphi}$. Note that the number of permutations determining $J_{\varphi}$ is at most $(2 c)+$ $(2 c)^{2}+\ldots+(2 c)^{\ell}<c^{\ell+1}$. Indeed, $c^{\ell+1}$ is an upper bound on the number of walks of length $\ell$ that could contain an edge of $\varphi$. Each such permutation itself could affect at most $c+\ldots+c^{\ell}<c^{\ell+1}$ events $J_{\psi}$. Thus, $\left|\mathcal{J}_{\varphi}\right| \leq\left(c^{\ell+1}\right)^{2}$.

We now apply the symmetric version of the Local Lemma. To that aim, we need to have that $\left(c^{\ell+1}\right)^{2} e^{-b / 100}<1 / 4$, which clearly holds since $\ell$ is fixed and $c$ is sufficiently large. Thus, by Lovász Local Lemma, $\mathbb{P}\left[\bigcap_{\forall \varphi} \overline{J_{\varphi}}\right]>0$. Thus, there exists pairings of the edges of the cones $\Gamma$ such that no event $J_{\varphi}$ occurs for every cone $\varphi$.

Let $H$ be the $\ell$-path-graph obtained from $\Gamma$ by removing all bad walks and short walks. Let $R:=G \backslash \underline{H}$. We can assume that $L$ is sufficiently large so that $\ell^{4} b<\varepsilon(1-\varepsilon) c / 2$. Then:

1. In every cone $\varphi$, there are no more than $\varepsilon c$ bad and short walks via it, so there are at least $(1-\varepsilon) c$ paths in $H$ via it. Hence, using the fact that $G$ is nearly equitably colored and by considering the special 1 -half and $\ell$-half cones, we obtain that for every vertex $v$, there are at least $\frac{1-\varepsilon}{2 \ell} d_{G}(v)$ paths in $H$ starting at $v$, and at least $\frac{1-\varepsilon}{2 \ell} d_{G}(v)$ paths in $H$ ending at $v$. Hence, $d_{H}(v) \geq \frac{1-\varepsilon}{\ell} d_{G}(v)$. The nearly equitable $\ell$-edge-coloring implies immediately that $d_{H}(v) \leq \frac{1+\varepsilon}{\ell} d_{G}(v)$.

2. For every pair of vertices $u, v, u \neq v$, among all walks via a cone of $u$, the ratio of walks going through $v$ is less than $\ell^{2} b / c<\varepsilon / 2 \ell$. Hence, among all walks via $u$, the ratio of walks going through $v$ is less than $\varepsilon / 2 \ell$. Thus

$$
\frac{|\{P \in \mathcal{P}: u, v \in P\}|}{d_{G}(u)} \leq \varepsilon / 2 \ell,
$$

and, hence, $\operatorname{conf}(u) \leq \varepsilon$. 
3. In every cone, there are no more than $\ell^{3} b$ bad and short walks via it, so the proportion of bad walks is at most $\ell^{3} b / c<\varepsilon(1-\varepsilon) / 2 \ell$. Hence, among all walks via a vertex $v$, the ratio of bad and short walks is less than $\varepsilon(1-\varepsilon) / 2 \ell$. Thus $d_{R}(v)<\varepsilon(1-\varepsilon) d_{G}(v) / 2 \ell$, implying $d_{R}(v) \leq \varepsilon d_{H}(v)$.

In the sequel, given two path-graphs $H_{1}$ and $H_{2}$ over a same graph, we will need to grow paths of, say, $H_{1}$ using the paths from $H_{2}$. This will essentially be achieved by considering every path $P$ of $H_{1}$, incident to, say, a vertex $v$, then considering a path $P^{\prime}$ incident to $v$ in $H_{2}$, and just concatenating $P$ and $P^{\prime}$. So that the concatenation can be performed this way for every path of $H_{1}$, we just need $H_{2}$ to have enough paths, and to make sure to evenly use these paths. The latter requirement can be ensured by just orienting $H_{2}$ in a balanced way, that is so that $\left|d^{+}(v)-d^{-}(v)\right| \leq 1$ for every vertex $v$, and choosing, as $P^{\prime}$, a path out-going from $v$. All such outgoing paths are called private paths of $v$ throughout the upcoming proofs.

The path-graph $H$ we get from $G$ after applying Theorem 3.3 hence satisfies $\frac{1-\varepsilon}{\ell} d_{G}(v) \leq d_{H}(v) \leq \frac{1+\varepsilon}{\ell} d_{G}(v)$ for every vertex $v$. If we preserve the orientation of the edges of $H$ as in the proof, and denote by $d_{H}^{+}(v)$ the number of paths starting from $v$ in $H$, we get

$$
\frac{1-\varepsilon}{2 \ell} d_{G}(v) \leq d_{H}^{+}(v) \leq \frac{1+\varepsilon}{2 \ell} d_{G}(v)
$$

for every vertex $v$. These $d_{H}^{+}(v)$ paths out-going from $v$ will hence be regarded as its private paths in what follows.

Theorem 3.4. Let $\ell$ be a positive integer, and $\varepsilon^{\prime}$ be a sufficiently small positive real number depending on $\ell$. There exists $L$ such that, for every graph $G$ with minimum degree at least $L$, there is an $(\ell, \ell+1)$-path-graph $H$ decomposing $G$ with

- $\operatorname{conf}(H) \leq 1 / 4(\ell+10)$, and

- $\frac{1-\varepsilon^{\prime}}{\ell} d_{G}(v) \leq d_{H}(v) \leq \frac{1+\varepsilon^{\prime}}{\ell} d_{G}(v)$ for every vertex $v$.

Proof. Let $\varepsilon^{\prime}>0$ be sufficiently small, and set $\varepsilon:=\varepsilon^{\prime} / 10 \ell$. Let $G_{1}$ be a $1 / 9 \ell$ fraction of $G$ obtained by Proposition 2.2, and $G_{2}:=G \backslash G_{1}$. By applying Theorem 3.3 on $G_{1}$ and $G_{2}$ with $\varepsilon$, we get two $\ell$-path-graphs $H_{1}$ and $H_{2}$ and two remainders $R_{1}$ and $R_{2}$ satisfying all properties from the statement of Theorem 3.3. For convenience, we will keep the orientation of the edges of $H_{1}$ and $H_{2}$ given by Theorem 3.3. Note that

$$
\frac{1-\varepsilon}{\ell} \cdot\left(\frac{d_{G}(v)}{9 \ell}-2\right) \leq d_{H_{1}}(v) \leq \frac{1+\varepsilon}{\ell} \cdot\left(\frac{d_{G}(v)}{9 \ell}+2\right)
$$

and

$$
\frac{1-\varepsilon}{\ell} \cdot\left(\frac{(9 \ell-1) d_{G}(v)}{9 \ell}-2\right) \leq d_{H_{2}}(v) \leq \frac{1+\varepsilon}{\ell} \cdot\left(\frac{(9 \ell-1) d_{G}(v)}{9 \ell}+2\right) .
$$


Now, we have $\frac{1-\varepsilon}{(1+\varepsilon)(9 \ell-1)} d_{H_{2}}(v)-10 \leq d_{H_{1}}(v) \leq \frac{1+\varepsilon}{(1-\varepsilon)(9 \ell-1)} d_{H_{2}}(v)+10$ for all vertices $v$. Let $R:=R_{1} \cup R_{2}$. Then for every vertex $v$, we have

$$
d_{R}(v)=d_{R_{1}}(v)+d_{R_{2}}(v) \leq \varepsilon d_{H_{1}}(v)+\varepsilon d_{H_{2}}(v) \leq 10 \ell \varepsilon d_{H_{1}}(v) .
$$

Arbitrarily orient the edges of $R$. In our construction, every step consists in extending an arc $v u$ of $R$ using a private (i.e., out-going) $\ell$-path starting at $v$ in $H_{1}$ that does not contain $u$ - thus forming an $(\ell+1)$-path. Since the conflict ratio of $H_{1}$ satisfies $\operatorname{conf}\left(H_{1}\right) \leq \varepsilon$, at most $\varepsilon d_{H_{1}}(v)$ paths in $H_{1}$ with $v$ as endpoint contain $u$. Note that the number of directed $\ell$-paths in $H_{1}$ starting at $v$ is $d_{H_{1}}^{+}(v) \geq \frac{1}{2} \cdot \frac{(1-\varepsilon) d_{G_{1}}(v)}{\ell}$. Thus, $d_{H_{1}}^{+}(v)-d_{R}(v)>\varepsilon d_{H_{1}}(v)$ since $L$ can be chosen sufficiently large. Hence, all the $d_{R}(v)$ edges can be used to form $(\ell+1)$-paths.

We call $H_{1}^{\prime}$ the resulting $(\ell, \ell+1)$-path-graph obtained by concatenating paths from $H_{1}$ and paths from $R$. Since $d_{R}(v) \leq 10 \ell \varepsilon d_{H_{1}}(v)$ for every $v$, the degree of $v$ in $H_{1}^{\prime}$ is as

$$
d_{H_{1}}(v)-10 \ell \varepsilon d_{H_{1}}(v) \leq d_{H_{1}^{\prime}}(v) \leq d_{H_{1}}(v)+10 \ell \varepsilon d_{H_{1}}(v) .
$$

Let $H:=H_{1}^{\prime} \cup H_{2}$. Then $H$ is an $(\ell, \ell+1)$-path-graph decomposing $G$, in which we have $d_{H}(v)=d_{H_{1}^{\prime}}(v)+d_{H_{2}}(v)$ for all vertices $v$. Thus,

$$
d_{H_{1}}(v)-10 \ell \varepsilon d_{H_{1}}(v)+d_{H_{2}}(v) \leq d_{H}(v) \leq d_{H_{1}}(v)+10 \ell \varepsilon d_{H_{1}}(v)+d_{H_{2}}(v) .
$$

Thus,

$$
\frac{1-\varepsilon}{\ell} d_{G}(v)-10 \ell \varepsilon d_{H_{1}}(v)+1 \leq d_{H}(v) \leq \frac{1+\varepsilon}{\ell} d_{G}(v)+10 \ell \varepsilon d_{H_{1}}(v)+1 .
$$

Since $\varepsilon^{\prime}=10 \ell \varepsilon$, we obtain that

$$
\frac{1-\varepsilon^{\prime}}{\ell} d_{G}(v) \leq d_{H}(v) \leq \frac{1+\varepsilon^{\prime}}{\ell} d_{G}(v) .
$$

Observe also that $d_{H_{1}^{\prime}}(v) / d_{H_{2}}(v) \leq 1 / 6 \ell$. Thus,

$$
\operatorname{conf}(H) \leq \operatorname{conf}\left(H_{2}\right)+\operatorname{conf}\left(H_{1}^{\prime}\right) / 6 \ell \leq \varepsilon+1 / 6 \ell<1 / 4(\ell+10),
$$

as required.

\section{Path-trees}

This part is the combinatorial core behind the proofs of our main results. We need here to show the existence of particular path-trees, namely $(\ell, 2 \ell)$-pathtrees, under mild connectivity and minimum degree requirements. These $(\ell, 2 \ell)$-path-trees will play a crucial role to insure that some path-graph has all of its vertices being of even degree. However, directly getting an $(\ell, 2 \ell)$ path-tree seems a bit challenging, and we will follow a long way for this, starting with a $(1,2)$-path-tree and making its paths grow.

We start off with the following lemma which is the key for the drop of the large edge-connectivity requirement. 
Lemma 4.1. Every 2-edge-connected multigraph has a subcubic spanning $(1,2)$-path-tree.

Proof. Let $G$ be connected and bridgeless. A structured-tree $T$ on $G$ is a strongly-connected digraph whose vertices are subsets $X_{i}$ of $V(G)$ satisfying the following properties:

- The $X_{i}$ 's form a partition of $V(G)$.

- The $\operatorname{arcs}$ of $T$ are of two types: the forward arcs forming a rooted outarborescence $A$, and the backward arcs, always directed from a vertex to one of its ancestors in $A$.

- Every arc $X_{i} X_{j}$ corresponds to some edge $x_{i} x_{j} \in E(G)$ such that $x_{i} \in X_{i}$ and $x_{j} \in X_{j}$.

- There is at most one backward arc leaving each vertex $X_{i}$ (unless $T$ is rooted at $X_{i}$ ).

- Internal vertices of $A$ are singletons.

- Every leaf $X_{i}$ of $A$ is spanned by a $(1,2)$-path-tree $T_{i}$ on $G$ with maximum degree 3 .

- The (unique) forward and backward arcs incident to a leaf $X_{i}$ have endpoints in $T_{i}$ with degree at most 2 , and if these endpoints coincide, the degree is at most 1 in $T_{i}$. In other words, adding the arcs as edges of $T_{i}$ preserves maximum degree 3 .

- Every edge of $G$ is involved in at most one $\operatorname{arc}$ of $T$ and one path of $T_{i}$. In other words, the edges of $G$ involved in $T$ and the $T_{i}$ 's are distinct.

We first show that $G$ has a structured-tree $T$, using a classical algorithm to find a strongly-connected orientation of a bridgeless graph. Fix a vertex $x$ and compute a Depth-First-Search tree $A$ from $x$. Orient the edges of $A$ from $x$ to form the forward arcs. By the DFS property, every edge of $G$ not in $A$ joins vertices which are parents. Orient these edges from the descendent to the ancestor: these are our backward arcs. Since we need to keep at most one backward arc issued from every vertex, we only keep the arc going to the lowest ancestor. Note that we obtain a structured-tree $T$, where each $X_{i}$ is a singleton vertex in $G$ and every leaf $T_{i}$ is a trivial $(1,2)$-path-tree on one vertex.

We now prove that every structured-tree $T$ with at least two vertices on $G$ can be reduced to one with less vertices. This will imply that $T$ can be reduced to a single vertex $X_{i}=V(G)$, hence providing the subcubic spanning $(1,2)$-path-tree $T_{i}$. 
We start by deleting the backward $\operatorname{arcs}$ of $T$ which are not needed for strong connectivity. Then we consider an internal vertex $X_{j}=\left\{x_{j}\right\}$ of $A$ with maximal height. Let $X_{1}, X_{2}, \ldots, X_{r}$ be the (leaf) children of $X_{j}$. Each forward $\operatorname{arc} X_{j} X_{i}$ corresponds to an edge $x_{j} x_{i}$, where $x_{j} \in X_{j}$ and $x_{i} \in X_{i}$. Each of these leaves $X_{i}$ is the origin of a backward $\operatorname{arc} X_{i} X_{i}^{\prime}$ which we write $y_{i} x_{i}^{\prime}$, where $y_{i} \in X_{i}$ and $x_{i}^{\prime} \in X_{i}^{\prime}$. We assume that our enumeration satisfies that $X_{i+1}^{\prime}$ is always an ancestor of $X_{i}^{\prime}$ (possibly equal to $X_{i}^{\prime}$ ). We now discuss the different reductions, in which the conditions of structured-trees are easily checked to be preserved.

- If $X_{j}$ has only one child $X_{1}$ and is not the origin of a backward arc, we merge $X_{1}$ and $X_{j}$ into a unique leaf $X_{1 j}$ spanned by the $(1,2)$-pathtree $T_{1} \cup\left\{x_{1} x_{j}\right\}$. If $X_{j}$ is the root, we are done, otherwise we let the forward arc entering $X_{1 j}$ be the one entering $X_{j}$, and the backward arc leaving $X_{1 j}$ be $X_{1 j} X_{1}^{\prime}$ (thus corresponding to the edge $y_{1} x_{1}^{\prime}$ ).

- If $X_{j}$ has only one child and is the origin of a backward $\operatorname{arc} X_{j} X_{j}^{\prime}$, we merge $X_{1}$ and $X_{j}$ into a unique leaf $X_{1 j}$ spanned by the $(1,2)$-pathtree $T_{1} \cup\left\{x_{1} x_{j}\right\}$. The forward arc entering $X_{1 j}$ is the one entering $X_{j}$, and the backward arc leaving $X_{1 j}$ is the one of $X_{j} X_{j}^{\prime}$.

- If $X_{j}$ has at least three children, or $X_{j}$ has two children and is the origin of a backward arc, observe that deleting $X_{1}$ and $X_{2}$ from $T$ preserves strong connectivity. Hence we merge $X_{1}$ and $X_{2}$ into a unique leaf $X_{12}$ spanned by the $(1,2)$-path-tree $T_{1} \cup T_{2} \cup\left\{x_{1} x_{j} x_{2}\right\}$. The forward arc entering $X_{12}$ is $x_{1}^{\prime} y_{1}$ (hence reversing the backward arc $X_{1} X_{1}^{\prime}$ ), and the backward arc leaving $X_{12}$ is $X_{12} X_{2}^{\prime}$ corresponding to $y_{2} x_{2}^{\prime}$.

- The last case is when $X_{j}$ has two children $X_{1}$ and $X_{2}$ and is not the origin of a backward arc. Here we merge $X_{1}, X_{2}, X_{j}$ into a unique leaf $X_{12 j}$ spanned by the (1,2)-path-tree $T_{1} \cup T_{2} \cup\left\{x_{1} x_{j}\right\} \cup\left\{x_{2} x_{j}\right\}$. If $X_{j}$ is the root, we are done, otherwise we let the forward arc entering $X_{12 j}$ be the one entering $X_{j}$, and the backward arc leaving $X_{12 j}$ be $X_{12 j} X_{2}^{\prime}$ (thus corresponding to $y_{2} x_{2}^{\prime}$ ).

We now turn our $(1,2)$-path-tree into a $(1, k)$-path-tree. For this we need to feed our original connected bridgeless graph $G$ (in which we find the subcubic $(1,2)$-path-tree) with some additional graph $H$, edge-disjoint from $G$, and with large enough degree.

Lemma 4.2. Let $G=(V, E)$ be a graph. Let $T$ be a spanning $(1, k)$-pathtree of $G$, where $k \geq 2$. Let $H$ be a graph on $V$, edge-disjoint from $G$, with the property that $d_{H}(v) \geq 2\left(d_{T}(v)+2 k\right)$ for every vertex $v$ of $G$. Then $G \cup H$ is spanned by a $(1, k+1)$-path-tree $T^{\prime}$. 
Proof. Start by arbitrarily orienting the edges of $H$ in a balanced way so that every vertex $v$ of $H$ has outdegree at least $d_{T}(v)+2 k$. Every vertex is hence provided with a set of private edges in $H$, namely, its out-going arcs. We will use these private edges to transform $k$-paths of $T$ into $(k+1)$-paths.

In this proof, a structured-tree $T^{\prime}$ on $G$ is a rooted $(1, k)$-path-tree whose vertices are subsets $X_{i}$ partitioning $V(G)$ and satisfying the following properties:

- If $X_{i} X_{j}$ is an edge in $T^{\prime}$, then there exists a corresponding 1-path or $k$-path $x_{i} x_{j} \in E(T)$, where $x_{i} \in X_{i}$ and $x_{j} \in X_{j}$.

- If $X_{j}$ has children $X_{1}, \ldots, X_{r}$ in $T^{\prime}$ then there is a unique $x_{j} \in X_{j}$ such that $x_{1} x_{j}, \ldots, x_{r} x_{j}$ are the corresponding paths in $E(T)$. We call $x_{j}$ the center of $X_{j}$.

- Every vertex $X_{i}$ of $T^{\prime}$ is spanned by a $(1, k+1)$-path-tree $T_{i}^{\prime}$.

Initially, let $T^{\prime}$ be the structured-tree $T$, where each $X_{i}$ is a singleton element $\left\{x_{i}\right\}$ in $V(T)$. Note that all the vertices of $T^{\prime}$ are trivial $(1, k+1)$ path-trees. Our goal is to iteratively reduce $T^{\prime}$ to a structured-tree consisting of one single vertex $X_{i}$, hence providing a spanning $(1, k+1)$-path-tree $T_{i}^{\prime}$. We will always make sure that at any iteration every center $x_{j}$ has at least $r+2 k$ private edges, where $r$ is the number of children of $X_{j}$, hence guaranteeing the repetition of the process. Let us now show that $T^{\prime}$ can be reduced to a structured-tree with less vertices (unless $T^{\prime}$ is a single vertex).

We consider an internal vertex $X_{j}$ of $T^{\prime}$ with maximal height. Let $X_{1}, \ldots, X_{r}$ be the (leaf) children of $X_{j}$ corresponding to paths $x_{1} x_{j}, \ldots, x_{r} x_{j}$, where $x_{j}$ is the center of $X_{j}$. If one of these paths, say $x_{1} x_{j}$, is an edge, we simply create a new vertex $X_{1 j}$ by concatenating $X_{1}$ and $X_{j}$ and letting $T_{1 j}^{\prime}=T_{1}^{\prime} \cup T_{j}^{\prime} \cup\left\{x_{1} x_{j}\right\}$. So we can assume that every $x_{i} x_{j}$-path has length $k$.

Consider $X_{j}$ and one of its children, say $X_{1}$. Let $y$ be a private neighbor of $x_{j}$ which is not a vertex of the path $x_{j} x_{1}$. Such a $y$ exists since $x_{j}$ has at least $2 k+r$ private neighbors. We distinguish two cases, in which the conditions of structured-trees are easily checked to be preserved:

- We first consider the case where $y$ is in some $X_{i}, X_{i} \neq X_{1}$. Call $P$ the $(k+1)$-path obtained by concatenating the $k$-path $x_{1} x_{j}$ with the edge $x_{j} y$. We here add $X_{1}$ to the set $X_{i}$ to form the set $X_{1 i}$ which is spanned by $T_{1 i}^{\prime}=T_{1}^{\prime} \cup T_{i}^{\prime} \cup\{P\}$. Here $x_{j}$ loses one private edge, but $X_{j}$ has one less child.

- The second case is when $y \in X_{1}$. We here add $X_{1}$ to the set $X_{j}$ to form the set $X_{1 j}$ which is spanned by the $(1, k+1)$-path-tree $T_{1 j}^{\prime}=$ $T_{1}^{\prime} \cup T_{j}^{\prime} \cup\left\{x_{j} y\right\}$. Here $x_{j}$ loses one private edge, but $X_{j}$ has one less child. 
The next result follows from Lemma 4.1 and repeated applications of Lemma 4.2:

Corollary 4.3. For every $\ell$, there exists $L$ such that if $G=(V, E)$ is a 2-edge-connected graph and $H$ is another graph on $V$, edge-disjoint from $G$, with minimum degree at least $L$, then one can form a spanning $(1, \ell+1)$ path-tree $T$ where $d_{T}(v) \leq d_{H}(v)$ for every vertex $v$.

Proof. We first apply Lemma 4.1 to get a subcubic (1,2)-path-tree $T_{0}$ from $G$. Fix a sufficiently small $\varepsilon_{1}>0$. We choose a sequence of edge-disjoint subgraphs $H_{1}, \ldots, H_{\ell-1}$ of $H$, where each $H_{i}$ is an $\varepsilon_{i}$-fraction of $H$, where $\varepsilon_{i+1}=4 \varepsilon_{i}$ for all $i$. Free to choose $L$ large enough as a function of $\varepsilon_{1}$, we can clearly obtain the desired subgraphs $H_{1}, \ldots, H_{\ell-1}$ by repeatedly applying Proposition 2.2. Since $L$ is sufficiently large, for each vertex $v$, we have that $d_{H_{1}}(v) \geq \varepsilon_{1} L-10 \ell^{\ell}>2 d_{T_{0}}(v)+4 \ell$. Thus, by Lemma 4.2 , we can use $H_{1}$ to extend $T_{0}$ into a $(1,3)$-path-tree $T_{1}$. Note that $d_{T_{1}}(v) \leq d_{T_{0}}(v)+d_{H_{1}}(v)$. Now we have that $d_{H_{2}}(v) \geq 3.5 d_{H_{1}}(v)>2 d_{T_{1}}(v)+4 \ell$, and thus, we can again use $H_{2}$ as an additional graph to extend $T_{1}$ into a $(1,4)$-path-tree $T_{2}$ with $d_{T_{2}}(v) \leq d_{T_{0}}(v)+d_{H_{1}}(v)+d_{H_{2}}(v)$. We iterate this process to form our $(1, \ell+1)$-path-tree $T$. Note that

$$
d_{T}(v) \leq d_{T_{0}}(v)+\sum_{i=1}^{\ell-1} d_{H_{i}}(v)<L \leq d_{H}(v),
$$

where the second to last inequality follows from the fact that we can choose $\varepsilon_{1}$ to be arbitrarily small.

Our ultimate goal now is to find path-trees where the lengths of the paths are a multiple of some fixed value $\ell$. One way to do so is to transform some $(1, \ell+1)$-path-trees into $(\ell, 2 \ell)$-path-trees. Note that if $\ell$ is even, and our graphs $G$ and $H$ are bipartite with the same bipartition, then there is no spanning $(\ell, 2 \ell)$-path-tree since an even path always connects a partite set with itself. The next result asserts that we can nevertheless connect each partite set separately.

Lemma 4.4. For every even integer $\ell$, there exists $L$ such that if $G=(V, E)$ is a 2-edge-connected bipartite graph with vertex partition $(A, B)$ and $H$ is another bipartite graph on $V$ with vertex partition $(A, B)$, edge-disjoint from $G$, and with minimum degree at least $L$, then one can form an $(\ell, 2 \ell)$-pathtree $T$ spanning $A$ where $d_{T}(v) \leq d_{H}(v)$ for every vertex $v$.

Proof. We first use a small $\varepsilon$-fraction of $H$ (and still call $H$ the graph minus this fraction for convenience) in order to apply Corollary 4.3. We can then obtain a spanning $(1, \ell+1)$-path-tree $T$ where $d_{T}(v) \leq \varepsilon d_{H}(v)$ for all vertices $v$. Note that $\varepsilon>0$ can be taken arbitrarily small since we can take $L$ so that $\varepsilon L$ is sufficiently large to apply Corollary 4.3. We now apply Theorem 3.3 on 
$H$ to find an $(\ell-1)$-path-graph $H^{\prime}$ (while preserving the balanced orientation given by the proof) on $H$ with $\operatorname{conf}\left(H^{\prime}\right) \leq \varepsilon$ and

$$
\frac{1-\varepsilon}{\ell-1} d_{H}(v) \leq d_{H^{\prime}}(v) \leq \frac{1+\varepsilon}{\ell-1} d_{H}(v)
$$

for all vertices $v$.

In our construction, every step consists in extending a path $P$ of $T$ starting at some vertex $v$ using a private (i.e., out-going) $(\ell-1)$-path from $H^{\prime}$. This will form either an $\ell$-path or a $2 \ell$-path. According to the conflict ratio assumption and the fact that $\varepsilon$ can be chosen to be sufficiently small, every such $P$ is conflicting with at most $|P| \varepsilon d_{H^{\prime}}(v)<d_{H^{\prime}}(v) / 8$ private paths of $v$, which is $1 / 4$ total number of private paths of $v$. In our upcoming process, the total number of private paths of $v$ we will use is at most $d_{T}(v) \leq$ $\varepsilon d_{H}(v)$, thus at most $1 / 4$ of the total number of private paths of $v$ since $d_{H^{\prime}}^{+}(v) \geq \frac{1-\varepsilon}{2 \ell} d_{H}(v)$. Hence, even if we have already used $1 / 4$ of the private paths of $v$, and we need a private path of $v$ which is non-conflicting with two paths of $T$ incident to $v$, we can still find one. Thus, in the upcoming arguments, we always assume that a private path is available whenever we need one.

We now turn to the construction of the $(\ell, 2 \ell)$-path-tree $T^{\prime}$ spanning $A$. A structured-tree $T^{\prime}$ on $G$ is a rooted tree in which the vertices are disjoint subsets $X_{i}$ whose union covers a subset of $V(G)$ containing $A$ with the following properties:

- If $X_{i} X_{j}$ is an edge in $T^{\prime}$, then there exists a corresponding 1-path or $(\ell+1)$-path $x_{i} x_{j} \in E(T)$, where $x_{i} \in X_{i}$ and $x_{j} \in X_{j}$.

- If $X_{j}$ has children $X_{1}, \ldots, X_{r}$ then there is a unique $x_{j} \in X_{j}$ such that $x_{1} x_{j}, \ldots, x_{r} x_{j}$ are the corresponding paths in $T$. We call $x_{j}$ the center of $X_{j}$

- Every vertex $X_{i}$ containing an element of $B$ is a singleton, i.e., $X_{i}=$ $\left\{x_{i}\right\}$.

- Every vertex $X_{i}$ of $T^{\prime}$ is spanned by an $(\ell, 2 \ell)$-path-tree $T_{i}^{\prime}$.

We again start with $T^{\prime}$ equal to $T$ in the sense that all $X_{i}^{\prime}$ 's are singletons, and all $T_{i}^{\prime}$ 's are trivial $(\ell, 2 \ell)$-path-trees. We root $T^{\prime}$ at some arbitrary vertex of $A$. Again our goal is to show that we can reduce $T^{\prime}$ until it is reduced to its root, which will therefore be equal to the set $A$, covered by an $(\ell, 2 \ell)$ path-tree. Note that since $\ell$ is even, we always have that an edge $X_{i} X_{j}$ of $T^{\prime}$ connects a vertex of $B$ and a subset of $A$.

Observe first that if $T^{\prime}$ has a leaf in $B$, we can simply delete it and keep our properties. We can then assume that all leaves are subsets of $A$. We consider an internal vertex $X_{j}$ of $T^{\prime}$ with maximal height. Let $X_{1}, X_{2}, \ldots, X_{r}$ 
be the (leaf) children of $X_{j}$ corresponding to the paths $x_{1} x_{j}, \ldots, x_{r} x_{j}$. Note that all $X_{i}$ 's are subsets of $A$, and that $X_{j}=\left\{x_{j}\right\}$ is in $B$. We now discuss the different reductions, in which the conditions of structured-trees are easily checked to be preserved.

Consider $X_{j}$ and one of its children, say $X_{1}$. Let $X_{k}$ be the parent of $X_{j}$ in $T^{\prime}$. Note that $X_{k}$ is a subset of $A$. We denote by $x_{j} x_{k}$ the path of $T$ joining $X_{j}$ and $X_{k}$. Let $y$ be a private neighbor of $x_{j}$ which is not a vertex of the path $x_{j} x_{k}$ and $x_{j} x_{1}$. We again consider two cases:

- First assume that $y$ is in some $X_{i}$, with $X_{i} \neq X_{1}$. We denote by $P^{\prime}$ the path obtained by concatenating the path $x_{1} x_{j}$ with $x_{j} y$. Note that $P^{\prime}$ is an $\ell$-path or a $2 \ell$-path. We add $X_{1}$ to the set $X_{i}$ to form the set $X_{1 i}$ which is spanned by $T_{1 i}^{\prime}=T_{1}^{\prime} \cup T_{i}^{\prime} \cup\left\{P^{\prime}\right\}$. Note that $x_{j}$ loses a private path, but $X_{j}$ has one less child.

- Otherwise, $y \in X_{1}$. We add $X_{1}$ to the set $X_{k}$ to form the set $X_{1 k}$ which is spanned by the $(\ell, 2 \ell)$-path-tree $T_{1 k}^{\prime}=T_{1}^{\prime} \cup T_{k}^{\prime} \cup\left\{P^{\prime \prime}\right\}$, where $P^{\prime \prime}$ is the concatenation of $x_{k} x_{j}$ and $x_{j} y$ (note that $P^{\prime \prime}$ is an $\ell$-path or a $2 \ell$-path). Note that $x_{j}$ loses a private path, but $X_{j}$ has one less child.

We will also need the following lemma.

Lemma 4.5. Let $\ell$ be a positive integer. There exists $L$ such that if $G_{1}=$ $(V, E)$ is a 2-edge-connected graph and $G_{2}=(V, F)$ is a graph of minimum degree at least $L$ edge-disjoint from $G_{1}$, then there is a connected $[\ell, \ell+3]$ path-graph $H$ decomposing $G_{1} \cup G_{2}$ with $\operatorname{conf}(H)<\frac{1}{2(\ell+10)}$.

Proof. Start by applying Lemma 4.1 to get a subcubic $(1,2)$-path-tree $T$ spanning $G_{1}$, and put the non-used edges of $G_{1}$ in $G_{2}$. Still calling this graph $G_{2}$, we decompose $G_{2}$ into a $1 /(5 \ell)$-fraction $R_{1}$ and a $1-1 /(5 \ell)$ fraction $R_{2}$, by Proposition 2.1. Thus, by Theorem 3.4, $G_{2}$ can then be decomposed into two $(\ell, \ell+1)$-path-graphs $H_{1}$ and $H_{2}$, respectively, both having conflict ratio at most $\frac{1}{4(\ell+10)}$, and verifying

$$
\frac{1-\varepsilon}{(5 \ell-1)(1+\varepsilon)} d_{H_{2}}(v) \leq d_{H_{1}}(v) \leq \frac{1+\varepsilon}{(5 \ell-1)(1-\varepsilon)} d_{H_{2}}(v)
$$

for all vertices $v$, and any $\varepsilon$.

In our construction, every step consists in extending a path $P$ of $T$ starting at $v$ using a private $\left(\geq \ell\right.$ )-path starting at $v$ in $H_{1}$ (where we recall that the private paths at any vertex are its out-going paths in a balanced orientation of $\left.H_{1}\right)$. This will form a $(\geq \ell)$-path. By the assumption on the conflict ratio, every $P$ is conflicting with at most, say, half of the private paths of $v$. Because $T$ is subcubic, the total number of private paths of $v$ we will need is at most 6 . Since $L$ can be chosen so that $\frac{L}{5 \ell} \cdot \frac{1}{2 \ell} \cdot\left(1-\frac{1}{4(\ell+10)}\right)$ 
is arbitrarily large, we can hence assume we have enough private paths for the whole process.

We now turn to the construction of the spanning $(\geq \ell)$-path-tree $T^{\prime}$ from $T$ and $H_{1}$. A structured-tree $T^{\prime}$ on $V$ is a rooted tree in which the vertices are disjoint subsets $X_{i}$ partitioning $V$ with the following properties:

- If $X_{i} X_{j}$ is an edge in $T^{\prime}$, then there exists a corresponding 1-path or 2-path $x_{i} x_{j} \in E(T)$, where $x_{i} \in X_{i}$ and $x_{j} \in X_{j}$.

- Every vertex $X_{i}$ of $T^{\prime}$ is spanned by a $(\geq \ell)$-path-tree $T_{i}^{\prime}$.

We again start with $T^{\prime}$ being equal to $T$ in the sense that all $X_{i}^{\prime}$ 's are singletons, and all $T_{i}^{\prime}$ 's are trivial $(\geq \ell)$-path-trees. We root $T^{\prime}$ at some arbitrary vertex. Again our goal is to show that we can reduce $T^{\prime}$ until it is reduced to its root, which will therefore be a spanning $(\geq \ell)$-path-tree.

We consider a leaf $X_{1}$ of $T^{\prime}$ with direct ancestor $X_{j}$. Then there exists a path $x_{1} x_{j}$ of $T^{\prime}$ having length 1 or 2 . We pick a private path $x_{j} y \in H_{1}$ not conflicting with the path $x_{1} x_{j}$. Assume $y \in X_{k}$. If $X_{k} \neq X_{1}$, then we denote by $P$ the path obtained by concatenating $x_{1} x_{j}$ and $x_{j} y$. Then we add $X_{1}$ to $X_{k}$ to form the set $X_{1 k}$ being spanned by $T_{1 k}^{\prime}=T_{1}^{\prime} \cup T_{k}^{\prime} \cup\{P\}$. If $X_{k}=X_{1}$, then we add $X_{1}$ to $X_{j}$ to form the set $X_{1 j}$ being spanned by $T_{1 j}^{\prime}=T_{1}^{\prime} \cup T_{j}^{\prime} \cup\left\{x_{j} y\right\}$. We choose a private path $x_{j} z$ in $H_{1}$ not conflicting with $x_{1} x_{j}$, and concatenate these two paths to get a path $x_{1} z$ that we put back into $H_{1}$.

Once the procedure above is finished, we end up with a spanning $(\geq \ell)$ path-tree $T^{\prime}$ and an $(\ell, \ell+1)$-path-graph $H_{1}^{\prime}$, where $H_{1}^{\prime}$ is the path-graph remaining from $H_{1}$ after we have used some of its paths to obtain $T^{\prime}$. Let $H:=T^{\prime} \cup H_{1}^{\prime} \cup H_{2}$. Then $H$ covers all edges of $G$. Note also that $H$ is an $[\ell, \ell+3]$-path-graph. Since $d_{T^{\prime} \cup H_{1}^{\prime}}(v) \leq d_{H_{1}}(v)+3$ for every vertex $v$ and we can choose $\epsilon$ to be sufficiently small, we have $d_{T^{\prime} \cup H_{1}^{\prime}}(v) \leq d_{H_{2}}(v) / 4(\ell+10)$ for every vertex $v$. Thus,

$\operatorname{conf}(H) \leq \operatorname{conf}\left(H_{2}\right)+\frac{\operatorname{conf}\left(T^{\prime} \cup H_{1}^{\prime}\right)}{4(\ell+10)}<\frac{1}{4(\ell+10)}+\frac{1}{4(\ell+10)} \leq \frac{1}{2(\ell+10)}$

which concludes the proof.

\section{Edge-partitioning a graph into $\ell$-paths}

We now have all ingredients to prove our main results, i.e., Theorems 1.3 and 1.4. We start off with the proof of Theorem 1.3.

Proof of Theorem 1.3. Without loss of generality, we assume that $\ell$ is even (as the statement for paths of length $2 k$ implies the statement for paths of length $k$ ). First of all, we consider a maximum cut $\left(V_{1}, V_{2}\right)$ of $G$, and just 
keep the set of edges $F$ across the cut. We call $G^{\prime}$ the graph $(V, F)$. Observe that $G^{\prime}$ is at least 12-edge-connected and has minimum degree at least $d_{\ell} / 2$.

By Proposition 2.4, there is an orientation $D$ of $G^{\prime}$ such that $D$ is 6 arc-strong and with $d^{+}(v)$ and $d^{-}(v)$ differing by at most 1 for every vertex $v$. By applying Proposition 2.5 to $D$ with some vertex $z$, we obtain 6 arcdisjoint out-arborescences, $T_{1}, \ldots, T_{6}$, rooted at $z$. Since each vertex $v$ has in-degree at most 1 in $T_{i}$ ( $z$ has in-degree 0$)$, and $d_{D}^{+}(v)$ and $d_{D}^{-}(v)$ differ by at most 1, the graph $T_{1} \cup \ldots \cup T_{6}$ is $1 / 2$-sparse in $G^{\prime}$.

Call now $G_{1}:=T_{1} \cup T_{2}, G_{2}:=T_{3} \cup T_{4}, G_{3}:=T_{5} \cup T_{6}$, and let $R$ be the graph consisting of all the edges of $F$ which are not in $G_{1}, G_{2}, G_{3}$. Observe that $G_{1}, G_{2}, G_{3}$ are connected and bridgeless. Furthermore, the graph $G_{1} \cup G_{2} \cup G_{3}$ is $1 / 2$-sparse in $G^{\prime}$, and hence $R$ is $1 / 2$-dense in $G^{\prime}$. In the sequel, several fractions of edges will be removed from $R$, but, for the sake of legibility, we will still call $R$ the set remaining after the transfers.

We turn $G_{1}$ into an $(\ell, 2 \ell)$-path-tree as follows: we consider a small $\varepsilon$ fraction $R_{1}$ of $R$, and apply Lemma 4.4 (with $G_{1}$ for $G$ and $R_{1}$ for $H$ ) to form an $(\ell, 2 \ell)$-path-tree $T^{\prime}$ spanning $V_{1}$ in which $d_{T^{\prime}}(v) \leq d_{R_{1}}(v)$ for all vertices $v \in V_{1}$. In other words, $T^{\prime}$ is $\varepsilon^{\prime}$-sparse in $R$ for some negligible $\varepsilon^{\prime}>0$ depending on $\varepsilon$. Similarly, we can obtain, from $G_{2}$, a $\varepsilon^{\prime}$-sparse $(\ell, 2 \ell)$ path-tree $T^{\prime \prime}$ spanning $V_{2}$. We still consider (neglecting the two $\varepsilon$-fractions) that $R$ is $1 / 2$-dense in $G^{\prime}$. Add all edges of $E(G) \backslash F$ to $R$.

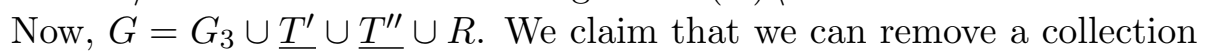
of $\ell$-paths or $2 \ell$-paths from the path-tree $T^{\prime}$ spanning $V_{1}$ in a way so that we can obtain that at most one vertex of $V_{1}$ has odd degree in $G$. Indeed, note that if $T$ is a tree and $X$ is an even subset of $V(T)$, then there exists a set of edges $F \subseteq E(T)$ such that for each vertex $x, d_{F}(x)$ is odd if and only if $x \in X$ (one way to see this is to note that the characteristic vector of $X$ is in the span of the incidence matrix of $T$ ). In particular, denoting by $X_{1}$ the set of all odd-degree vertices of $G_{3} \cup T^{\prime \prime} \cup R$ inside $V_{1}$ (and possibly removing one vertex of $X_{1}$ to make $X_{1}$ of even size) we can find a subtree $F^{\prime}$ of $T^{\prime}$ such that $d_{F^{\prime}}(v)$ is odd if and only if $v \in X_{1}$. In other words, removing the $\ell$ - or $2 \ell$-paths of $T^{\prime}$ corresponding to $F^{\prime}$ leaves $G$ with every vertex of $V_{1}$ (except possibly one) having even degree. Similarly, we remove paths of the path-tree $T^{\prime \prime}$ spanning $V_{2}$ so that at most one vertex of $V_{2}$ has odd degree.

We still call $G$ the remaining graph after the procedure, and we add the remaining edges of $\underline{T^{\prime}} \cup \underline{T^{\prime \prime}}$ to $R$. Then $G=G_{3} \cup R$. Note that $G_{3}$ is 2edge-connected, and $R$ is $1 / 4$-dense in $G$. By applying Lemma 4.5 (with $G_{3}$ for $G_{1}$ and $R$ for $G_{2}$ ), we can express $G$ as a connected [ $\left.\ell, \ell+3\right]$-path-graph $H$ with $\operatorname{conf}(H)<1 / 2(\ell+10)$. Note that $d_{G}(v)-d_{H}(v)$ is even for every vertex $v$ - so the degree of every vertex in $H$ is even, except (possibly) for two vertices $v_{1} \in V_{1}$ and $v_{2} \in V_{2}$. In this case, we add a dummy $\ell$-path from $v_{1}$ to $v_{2}$ in $H$ to make $H$ eulerian. By Theorem 3.2, we get that $H$ has a non-conflicting eulerian tour from which we can deduce the desired decomposition. 
One important fact in the proof of Theorem 1.3 is that, when constructed, the path-graph $H$ covers all edges of $G$. For this reason, it should be clear that the parity of the degree of every vertex is preserved from $G$ to $H$. This simple remark implies the following interesting counterpart result on eulerian graphs that are sufficiently edge-connected and have large enough minimum degree.

Theorem 5.1. For every integer $\ell$, there exists $d_{\ell}$ such that every 4-edgeconnected eulerian graph $G$ with minimum degree at least $d_{\ell}$ has an eulerian tour with no cycle of length at most $\ell$.

Proof. Following the arguments in the second paragraph of Theorem 1.3, we can extract from $G$ two trees $T_{1}$ and $T_{2}$ so that $T_{1} \cup T_{2}$ is $1 / 2$-sparse in $G$. Let $G_{1}:=T_{1} \cup T_{2}$, and $G_{2}:=G \backslash G_{1}$. Then $G_{1}$ is 2-edge-connected, and $G_{2}$ is $1 / 2$-dense. Applying Lemma 4.5 , we can express $G$ as a connected $[\ell, \ell+3]$-path-graph $H$ with $\operatorname{conf}(H)<1 / 2(\ell+10)$. Since $G$ is eulerian, so is $H$. Hence $H$ has non-conflicting eulerian tours according to Theorem 3.2, and these tours do not have cycles of length at most $\ell$ since all paths of $H$ have length at least $\ell$.

Theorem 5.1 now directly implies Theorem 1.4.

\section{References}

[1] J. Bensmail, A. Harutyunyan, T.-N. Le, M. Merker and S. Thomassé. A Proof of the Barát-Thomassen Conjecture. Journal of Combinatorial Theory, Series B, 124:39-55, 2017.

[2] F. Botler, G.O. Mota, M. Oshiro and Y. Wakabayashi. Decomposing highly edge-connected graphs into paths of any given length. Journal of Combinatorial Theory, Series B, 122:508-542, 2017.

[3] F. Botler, G.O. Mota, M. Oshiro and Y. Wakabayashi. Decompositions of highly connected graphs into paths of length five. Discrete Applied Mathematics, Doi: 10.1016/j.dam.2016.08.001, 2016.

[4] J. Barát and C. Thomassen. Claw-decompositions and Tutteorientations. Journal of Graph Theory, 52:135-146, 2006.

[5] J. Edmonds, Edge-disjoint branchings. B. Rustin, editor, Combinatorial Algorithms, pp. 91-96, Academic Press, 1973.

[6] B. Jackson, On circuit covers, circuit decompositions and Euler tours of graph, Surveys in Combinatorics, London Mathematical Society Lecture Note Series, pp. 191-210, 187: 1993. 
[7] C. McDiarmid. Concentration for Independent Permutations. Combinatorics, Probability and Computing, 11:163-178, 2002.

[8] M. Molloy and B. Reed. Graph Colouring and the Probabilistic Method. Springer, 2002.

[9] C.St.J.A. Nash-Williams. On orientations, connectivity and odd-vertexpairings in finite graphs. Canadian Journal of Mathematics, 12:555-567, 1960.

[10] M. Stiebitz, D. Scheide, B. Toft, L. M. Favrholdt. Graph Edge Coloring: Vizing's Theorem and Goldberg's Conjecture. Wiley, 2012.

[11] C. Thomassen. Decompositions of highly connected graphs into paths of length 3. Journal of Graph Theory, 58:286-292, 2008.

[12] C. Thomassen. Edge-decompositions of highly connected graphs. Abhandlungen aus dem Mathematischen Seminar der Universität Hamburg, 18:17-26, 2008.

[13] C. Thomassen. Decomposing graphs into paths of fixed length. Combinatorica, 33(1):97-123, 2013. 